\title{
Liver Segmentation Using Automatically Defined Patient Specific B-Spline Surface Models
}

\author{
Yi Song, Andy J. Bulpitt, and Ken W. Brodlie \\ School of Computing, University of Leeds, UK \\ \{scsys, A.J.Bulpitt, K.W.Brodlie\} aleeds.ac.uk
}

\begin{abstract}
This paper presents a novel liver segmentation algorithm. This is a model-driven approach; however, unlike previous techniques which use a statistical model obtained from a training set, we initialize patient-specific models directly from their own pre-segmentation. As a result, the non-trivial problems such as landmark correspondences, model registration etc. can be avoided. Moreover, by dividing the liver region into three sub-regions, we convert the problem of building one complex shape model into constructing three much simpler models, which can be fitted independently, greatly improving the computation efficiency. A robust graph-based narrow band optimal surface fitting scheme is also presented. The proposed approach is evaluated on 35 CT images. Compared to contemporary approaches, our approach has no training requirement and requires significantly less processing time, with an RMS error of $2.44 \pm 0.53 \mathrm{~mm}$ against manual segmentation.
\end{abstract}

\section{Introduction}

This work forms part of a project to develop virtual environments for training in interventional radiological procedures. It requires major abdominal structures, e.g. liver, kidney and blood vessels etc, to be segmented with particular interest in those cases where typical pathology is presented. The data for liver segmentation in this study therefore comes from patients with various pathologies and is obtained from different sources using different protocols which vary in quality and resolution and include both contrast enhanced and non-enhanced data. These diversities increase the variability of the liver data in both shape and texture.

Many techniques for liver segmentation have been proposed and implemented in recent years, see [1] for a recent review. These can be classified as texture-based and model-driven approaches. Due to the similar intensity values of some surrounding structures in CT data, approaches which are mainly based on local intensity or intensity gradient features are usually not sufficient to differentiate liver tissue. Therefore, model-based approaches have been widely explored where prior knowledge about the typical shape of a liver is used to constrain the segmentation process. Despite a number of different representations $[2,3,4]$, many of these approaches rely on principal component analysis of corresponding landmark points marked on a training set to calculate allowed modes of variation of the shape model which may result in limited deformations impeding the exact adaptation to the structure of interest. Although 
techniques [5,6] have been developed to overcome this, these approaches still require the shape model to be roughly aligned and oriented to the structure of interest so that the iterative search procedure can lock onto the target.

In this paper, a novel shape model construction method is presented. Unlike previous work utilizing training datasets to capture the mean modes of shape variations, our patient-specific shape model is directly derived from each image dataset. Our previous work [7] has shown how a target region can be captured through applying morphological erosion on an edge enhanced image followed by a region growing algorithm (Fig. 1a); this pre-segmentation is then automatically embedded into a curvature-driven level set to evolve a smooth surface toward the real boundary. Due to the similarity of intensity values with surrounding structures, the liver presegmentation is likely to include some non-liver tissues (Fig. 1b). Hence in this paper, we construct a three-patch surface model (Fig. 1c) to eliminate such unwanted parts from the pre-segmentation. Only the most reliable information from the presegmentation is used to initialize our three-patch shape model, representing upper, right lobe and lower liver boundaries. A graph-based optimal surface fitting scheme is then applied independently on each patch (Fig. 1e), from which we obtain a refined pre-segmentation result having non-liver tissues removed (Fig. 1d).
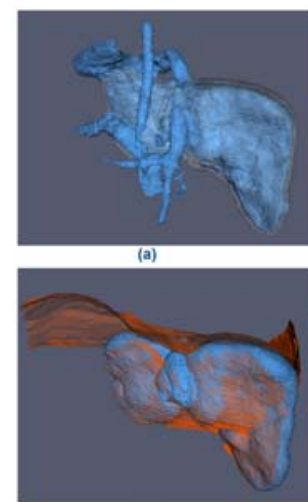

(d)

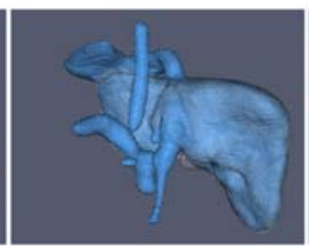

(b)

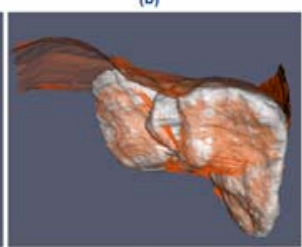

(e)

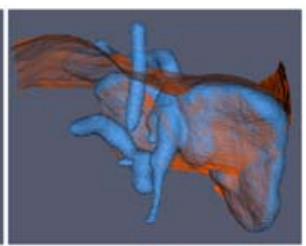

(c)

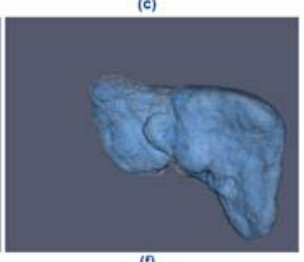

(f)

Fig. 1. Model construction ${ }^{1}$. (a) Initial liver estimation (blue) with manual segmentation (white). (b) Pre-segmentation (blue) with manual segmentation (white). (c) Deformed surface patches (orange) on the pre-segmentation (blue). (d) Refined pre-segmentation after non-liver tissues are removed. (e) Manual segmentation with surface patches (orange), for comparison with (d). (f) Final result of liver segmentation (blue) with the manual segmentation (white).

\section{Method}

The method consists of the following three main steps:

1) Liver pre-segmentation. This is obtained by applying our previous work [7] followed by a smoothness operation using a curvature-driven level set approach. The liver pre-segmentation serves as a basis for the subsequent segmentation.

\footnotetext{
${ }^{1}$ All the cases illustrated in this paper are from the datasets provided by [8].
} 
2) Model initialization and deformation. This is a fully automatic process to remove unwanted tissues from the pre-segmentation. Firstly a three-patch surface model is initialized, representing the upper, right lobe and lower liver surfaces, separately (section 2.1). Next a graph-based optimal surface fitting scheme (section 2.2) is applied to recover the "real" liver boundaries (Fig. 1c,1e), from which we can obtain a refined pre-segmentation result with non-liver tissues removed (Fig. 1d).

3) Liver pre-segmentation refinement and precise liver region recovery by level set evolution (section 2.3). To recover some missing parts, such as some tips or small perturbations of the liver edges, due to morphological and smoothness operations in step one, we re-implement the level set evolution but driven by both image force and inner force, to obtain a more accurate liver contour (Fig. 1f).

\subsection{Model Initialization}

The feature points for the three-patch surface construction are identified on each coronal slice of the pre-segmentation images. Due to the property of B-Splines, the sensitivity of the surface to some poorly located feature points is reduced when it is initialized from a large number of points.

\subsubsection{Upper Liver Surface Construction}

The upper liver surface aims to separate liver and heart regions by using a curved surface approximating the base of the right and left lungs.

Initially, the lungs are segmented using region growing, seeded automatically by finding points with the lowest HU value directly above the highest part of the liver right lobe found from the pre-segmentation. From the segmentations the corner points on the bottom of each lung (Fig. 2a) are detected automatically on every coronal slice and a number of points sampled between the left and right corners of right/left lung along large gradient values. The number of sampled points is determined by the distance between the left and right bottom corners of the right and left lung. The B-Spline reconstruction technique [9] is applied to create the curved surface approximating the set of sample points (Fig. 2).
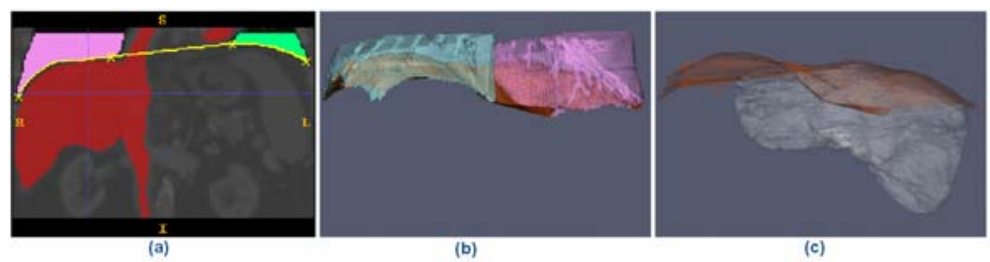

Fig. 2. Upper liver surface construction. (a) Feature points of lungs and B-Spline reconstruction (2D). (b) Constructed upper liver surface (orange) fitting to the bottom of the lungs (3D). (c) Upper liver surface is overlapped on the manual segmentation result (white).

\subsubsection{Liver Right Lobe Boundary Construction}

The liver right lobe boundary is created to delineate the abdominal cavity wall even when it is only partially detectable on the image. The initialized curved surface 
encapsulates the right lung wall ${ }^{2}$ and the right lobe of the liver pre-segmentation (Fig. 3b, 4b). A point set for B-Spline interpolation is created by sampling points in each coronal slice. For the lung wall, three evenly spaced points are selected. For feature points on the liver right lobe wall, to avoid noise introduced to the presegmentation, only two points located close to the bottom of the lung and one point located at the inferior segment of the right lobe (Fig. 3a) are used.

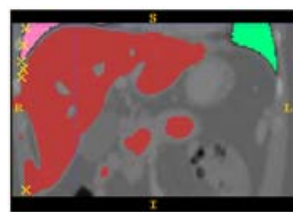

(a)

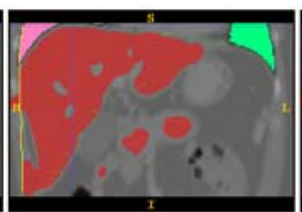

(b)

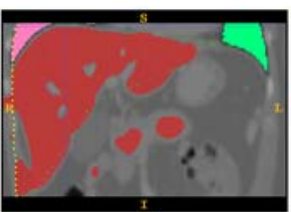

(c)

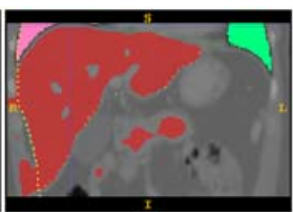

(d)

Fig. 3. Liver right lobe boundary (yellow) initialization and deformation (2D). (a) Feature points (yellow). (b) B-Spline reconstruction. (c) Discretization. (d) Deformation.

The initialization and deformation result (3D) of the liver right lobe wall is also shown in Fig. 4. For comparison, we overlay the fitted boundary onto the manual segmentation result in Fig. 4d. More detailed discussion on the deformation procedure is given in section 2.2 .

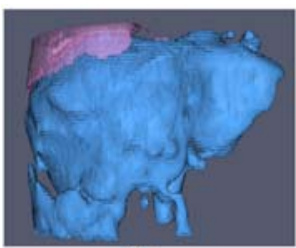

(a)

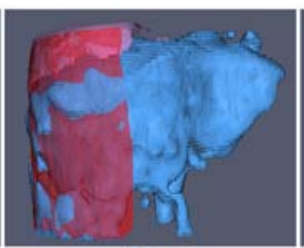

(b)

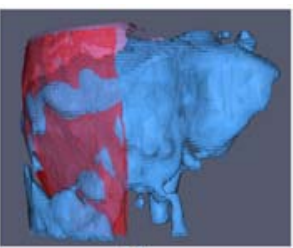

(c)

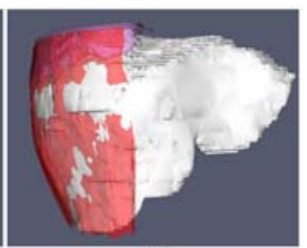

(d)

Fig. 4. Liver right lobe boundary initialization and deformation (3D). (a) Liver pre-segmentation (blue) and right lung detection (pink). (b) Liver right lobe boundary initialization (red). (c) Liver right lobe boundary deformation (fitting). (d) Deformed liver right lobe boundary (red) overlays on the manual segmentation result (white).

\subsubsection{Lower Liver Surface Construction}

The objective of approximating the lower liver surface is to exclude any non-liver tissues under the liver. The main problematic area in our pre-segmentation result is the portal vein, which is located near the centre of the liver. Therefore, we choose two pairs of sample points at both sides of the bottom of the liver, 1) the leftmost point on the left lobe boundary and a second point on the left lobe boundary but $7 \mathrm{~mm}$ under the first, 2) the lowest point on the inferior segment of the right lobe and a point which is $7 \mathrm{~mm}$ above (Fig. 5a). The distance is only used as a reference to obtain the second point in each pair. This distance is small enough to ensure the initialized liver lower patch excludes the portal vein. In a similar manner to the other surfaces, the lower liver surface is created from the sample points by using B-Spline reconstruction (Fig. 5b).

${ }^{2}$ The right lung always exists for liver segmentation where the top of the liver right lobe is included in image sources. 


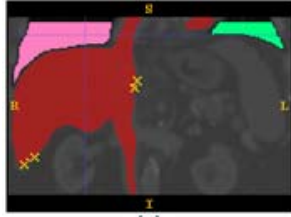

(a)

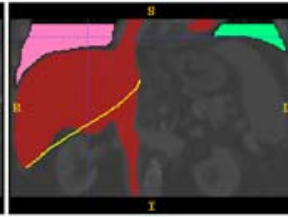

(b)

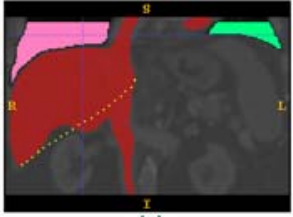

(c)

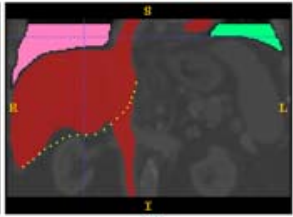

(d)

Fig. 5. Lower liver surface (yellow) initialization and deformation (2D). (a) Feature points (yellow). (b) B-Spline reconstruction. (c) Discretisation. (d) Deformation.

\subsection{Deformable Model}

Similar to the work presented in [5,10], the initial liver model (Fig. 6a) is deformed to create the "real" liver boundary (Fig. 6d) by applying a graph based fitting scheme. The details of constructing the directed graph can be found in [10]. A simplified visualization of this graph structure is given in Fig. 6c. To increase computation efficiency and robustness to outliers, we introduce the narrow band concept of the level set method. The lower liver surface is used to illustrate our approach.

According to the property of the B-Spline, the continuous lower liver surface can be discretised at any desired resolution [9]. In practice, to increase robustness to the local minimum, we adopt a sampling scheme where an average distance between adjacent vertices is about 3 times the voxel size of the original image (Fig. 3c,5c). We denote the discretised lower liver surface as $\boldsymbol{S}_{\mathrm{t}=0}=\left(\boldsymbol{V}_{0}, \boldsymbol{E}\right)$ with vertices set $\boldsymbol{V}_{0}$ and edges set $\boldsymbol{E}$. The graph search determines the optimal position $v_{\mathrm{i}}^{*}$ for each vertex $v_{\mathrm{i}} \in$ $\boldsymbol{V}_{0}$. The final optimized surface is denoted as $\boldsymbol{S}^{*}=\left(\boldsymbol{V}^{*}, \boldsymbol{E}\right)$. The vertex at any location can then be derived using B-Spline interpolation.

The external force is computed from the edge map of the pre-segmentation image, which serves as a template eliminating any region outside the liver region (Fig. 6b). The region inside the liver pre-segmentation is denoted by $\boldsymbol{R}_{\mathrm{ps}}$. Defining an infinite line $\boldsymbol{L}\left(v_{\mathrm{i}}\right)$ starting at vertex $v_{\mathrm{i}}$ with the direction $\boldsymbol{N}\left(v_{\mathrm{i}}, t\right), \boldsymbol{L}\left(v_{\mathrm{i}}\right)$ intersects with an edge on the edge map at point $\mathrm{p}_{\mathrm{i}}{ }^{*}$. A spring force drives the vertex $v_{\mathrm{i}}$ in the corresponding direction:

$$
F_{e x t}\left(v_{i}\right)=\left\{\begin{array}{cc}
\delta & \text { if }\left|p_{i}^{*}-v_{i}\right|>\delta \\
\left|p_{i}^{*}-v_{i}\right| & \text { if }\left|p_{i}^{*}-v_{i}\right| \leq \delta \\
0 & v_{i} \notin R_{p s}
\end{array}\right.
$$

$\delta$ is the width of the narrow band around $S_{\mathrm{t}}$. A smoothness constraint, denoted by $\Delta$, is imposed on the geometric relations of the nodes in the graph. That is, a shift between any pair of neighbouring points on each sought surface cannot be bigger than $\Delta$. A smaller value of $\Delta$ forces the sought surface to be smoother.

The surface fitting scheme is implemented in an iterative way. The process is stopped when either the predefined number of iterations has been achieved or the average vertex movement falls below a given threshold. More example results of deformation have been depicted in Fig. 3(5)d (2D) and Fig. 4c (3D). 


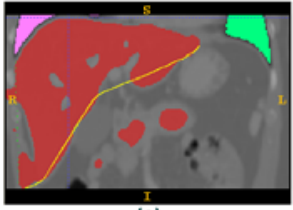

(a)

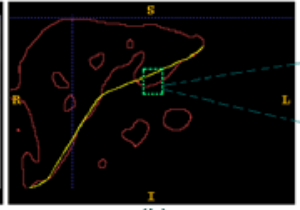

(b)

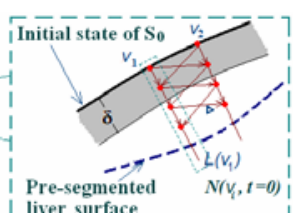

(c)

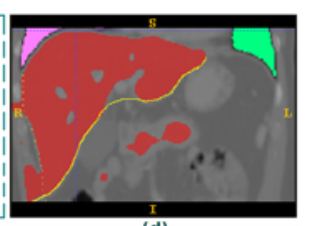

(d)

Fig. 6. Deformable model (patient-2). (a) Model initialization. (b) Edge map. (c) Graph. (d) Model deformation.

\subsection{Pre-segmentation Refinement and Precise Liver Segmentation}

Using the three optimized surface patches, the tissues outside the boundary (indicated by the surface normal direction) can be removed from the pre-segmentation (Fig. 1d). The result is a refined liver pre-segmentation which is the input of the level set evolution for accurate liver shape recovery (automatically).

The level set evolution in this section is driven by a joint force, i.e. image based force (external force) and curvature force (internal force), where the image based force is dominant. The external force is computed from a probabilistic map [11].

As the refined estimation is close to the real boundary, only a few iterations are required and thus the final result is not sensitive to the choice of parameters. We use 0.2 and 0.8 , corresponding to the weights of curvature and image force respectively, for all experiments.

\section{Evaluation and Experimental Results}

In our project, it is desirable to segment liver inner structures, e.g. tumors and vessels, as separate objects. For comparison, we automatically integrate the explicitly segmented inner structures into the liver segmentation (Fig. 7b). The surface patches constructed in section 2 are used to trim vessels outside the liver region (Fig. 7a).

\subsection{Evaluation of Accuracy}

Our approach has been evaluated on 20 patient $\mathrm{CT}$ datasets ${ }^{3}$ provided by the organizers of the MICCAI Workshop on 3D Segmentation in the Clinic [8] and 5 further patient CT data from the $\mathrm{CRaIVE}^{4}$ project. Both volume-based and mesh-based evaluations are conducted. Manual segmentations are taken as references.

\section{1) Volume comparison.}

This is measured based on three criteria defined by [12], which are all expressed as a fraction of the volume of reference models; 1) True positive volume fraction (TPVF): the fraction of voxels in the intersection of our segmentation and the reference model; 2) False negative volume fraction (FNVF): the fraction of voxels defined in manual segmentation but missed by our method; 3) False positive volume fraction (FPVF): the fraction of voxels falsely identified by our method. The average TPVF is

\footnotetext{
${ }^{3}$ Since there is no training process required by our method, we use their 20 training datasets for testing as well. The results of 10 test datasets have been submitted to [8] for evaluation.

${ }^{4}$ Collaborators in Radiological Interventional Virtual Environments, http://www.craive.org.uk
} 
95.77( \pm 2.07$) \%$. FNVF and FPVF are 4.23( \pm 2.07$) \%$ and 4.31( \pm 2.49$) \%$, respectively.. To be comparable with other liver segmentation experiments, we also calculate the overlap error and volume difference (Table 1).

\section{2) Mesh comparison}

This experiment is based on the distance measurement between vertices of the reference model and our result. The mesh is created by using the marching cubes algorithm from the VTK library, using the same parameters for all datasets. The average RMS error is $2.44 \pm 0.53 \mathrm{~mm}$. The average maximum distance $16.84 \pm 4.35 \mathrm{~mm}$ and the average mean distance is $-0.15 \pm 0.22 \mathrm{~mm}$.

\subsection{Evaluation of Efficiency}

Our method was performed on an Intel Core2 $2.66 \mathrm{GHz}$ processor. The average segmentation time is about 1 minute (step1: 15sec.; step2: 25-30sec.; step3: 10sec.). The comparison to recent liver segmentation experiments is given in Table 1.

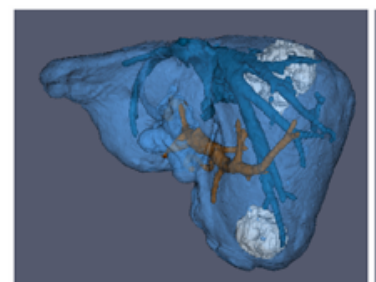

(a)

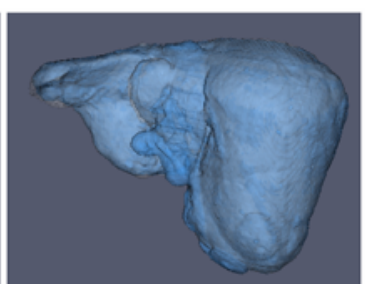

(b)

Fig. 7. (a) Liver Segmentation (light blue), tumor (white) and vessels (dark blue and brown). (b) Segmentation after merging (in blue), overlapped with the manual segmentation (white).

Table 1. Comparison to recent liver segmentation experiments. They are listed in the order of the best automatic, fastest automatic and best semi-automatic methods.

\begin{tabular}{|c|c|c|c|c|c|c|c|c|}
\hline Method & $\begin{array}{c}\text { Overlap Error } \\
{[\%]}\end{array}$ & $\begin{array}{c}\text { Volume Diff. } \\
{[\%]}\end{array}$ & $\begin{array}{c}\text { Avg. Dist. } \\
{[\mathrm{mm}]}\end{array}$ & $\begin{array}{c}\text { RMS Dist. } \\
{[\mathrm{mm}]}\end{array}$ & $\begin{array}{c}\text { Max. Dist. } \\
{[\mathrm{mm}]}\end{array}$ & Run time & $\begin{array}{c}\text { Datasets } \\
\text { tested }\end{array}$ & contrast \\
\hline Kainmueller [13] & 7.0 & -3.6 & 1.1 & 2.3 & 20.9 & $15 \mathrm{mins}$ & 10 & yes \\
\hline Rusko [14] & 10.7 & -4.3 & 1.8 & 3.8 & 28.3 & $56 \mathrm{sec}$. & 10 & yes \\
\hline Lee et al. [15] & 6.9 & -1.3 & 1.1 & 2.1 & 21.3 & $7.4 \mathrm{mins}$ & 10 & yes \\
\hline Our approach & 8.15 & 0.079 & -0.15 & 2.44 & 16.84 & $1 \mathrm{~min}$ & 25 & mixed \\
\hline
\end{tabular}

\section{Conclusions and Future Work}

Despite a large body of literature, (semi-) automatic liver segmentation from a 3D volume remains a challenge. Due to the large variations in shape and intensity pattern, the success of the classic statistical model-based approaches is often compromised by the limited number of training datasets. To overcome this, we propose a novel model-driven approach which creates a deformable model from each patient dataset directly. Moreover, by converting the problem of building one complex shape model into constructing three much simpler models that can be fitted independently, we greatly improve the computation efficiency. 
Acknowledgments. This work is funded by the UK EPSRC (EP/E002749). The authors would like to thank Dr F. Bello and Dr P. Villard from Imperial College for help with evaluation. We would also like to thank Dr D. Gould at University of Liverpool for data acquisition and valuable clinical advice.

\section{References}

1. Campadelli, P., Casiraghi, E.: Liver Segmentation from CT Scans: A Survey. In: Masulli, F., Mitra, S., Pasi, G. (eds.) WILF 2007. LNCS (LNAI), vol. 4578, pp. 520-528. Springer, Heidelberg (2007)

2. Florin, C., Paragios, N., Funka-Lea, G., Williams, J.: Liver Segmentation Using Sparse 3D Prior Models with Optimal Data Support. In: Karssemeijer, N., Lelieveldt, B. (eds.) IPMI 2007. LNCS, vol. 4584, pp. 38-49. Springer, Heidelberg (2007)

3. Ling, H., Zhou, S.K., Zheng, Y., et al.: Hierarchical, Learning-based Automatic Liver Segmentation. In: IEEE conf. on Computer Vision and Pattern Recognition, pp. 1-8 (2008)

4. Wimmer, A., Soza, G., Hornegger, J.: Two-stage Semi-automatic Organ Segmentation Framework Using Radial Basis Functions and Level Set. In: Heimann, T., Styner, M., van Ginneken, B. (eds.) MICCAI workshop on 3D Segmentation in the Clinic, pp. 179-188 (2007)

5. Heimann, T., Meinzer, H., Wolf, I.: A Statistical Deformable model for the Segmentation of Liver CT Volumes. In: MICCAI workshop on 3D Segmentation in the Clinic (SLIVER 2007), pp. 161-166 (2007)

6. Lamecker, H., Lange, T., Seebass, M.: A Statistical Shape Model for the Liver. In: Dohi, T., Kikinis, R. (eds.) MICCAI 2002. LNCS, vol. 2489, pp. 421-427. Springer, Heidelberg (2002)

7. Song, Y., Bulpitt, A., Brodlie, K.: Efficient Semi-automatic Segmentation for Creating Patient Specific Models for Virtual Environments. In: MICCAI workshop CVII, pp. 22-34 (2008)

8. Heimann, T., van Ginneken, B., Styner, M., et al.: Comparison and Evaluation of Methods for Liver Segmentation from CT datasets. J. IEEE Trans. on Medical Imaging (2009) (in press) doi:10.1109/TMI.2009.2013851

9. Song, Y., Bai, L.: 3D Modeling for Deformable Objects. In: Perales, F.J., Fisher, R.B. (eds.) AMDO 2008. LNCS, vol. 5098, pp. 175-187. Springer, Heidelberg (2008)

10. Li, K., Millington, S., Wu, X., Chen, D., Sonka, M.: Simultaneous segmentation of multiple closed surfaces using optimal graph searching. In: Christensen, G.E., Sonka, M. (eds.) IPMI 2005. LNCS, vol. 3565, pp. 406-417. Springer, Heidelberg (2005)

11. Zhu, S.C., Yuille, A.: Region Competition: Unifying Snakes, Region Growing, and Bayes/MDL for Multiband Image Segmentation. J. IEEE Trans. PAMI 18, 884-900 (1996)

12. Udupa, J.K., Leblanc, V.R., Schmidt, H., et al.: A Methodology for Evaluating Image Segmentation Algorithm. In: SPIE, vol. 4684, pp. 266-277 (2002)

13. Kainmueller, D., Lange, T., Lamecker, H.: Shape Constrained Automatic Segmentation of the Liver based on a Heuristic Intensity Model. In: Heimann, T., Styner, M., van Ginneken, B. (eds.) MICCAI workshop on 3D Segmentation in the Clinic, pp. 109-116 (2007)

14. Rusko, L., Bekes, G., Nemeth, G., Fidrich, M.: Fully Automatic Liver Segmentation for Contrast-enhanced CT images. In: Heimann, T., Styner, M., van Ginneken, B. (eds.) MICCAI workshop on 3D Segmentation in the Clinic, pp. 143-150 (2007)

15. Lee, J., Kim, N., Lee, H., et al.: Efficient Liver Segmentation Exploiting Level-set Speed Images with 2.5D Shape Porpagation. In: Heimann, T., Styner, M., van Ginneken, B. (eds.) MICCAI workshop on 3D Segmentation in the Clinic, pp. 189-196 (2007) 